\title{
DUALITY OF MEASURE AND CATEGORY IN INFINITE-DIMENSIONAL SEPARABLE HILBERT SPACE $\ell_{2}$
}

\author{
GOGI PANTSULAIA
}

Received 12 March 2001 and in revised form 16 August 2001

We prove that an analogy of the Oxtoby duality principle is not valid for the concrete nontrivial $\sigma$-finite Borel invariant measure and the Baire category in the classical Hilbert space $\ell_{2}$.

2000 Mathematics Subject Classification: 28A35, 28C15, 28C20, 54E52.

As usual, we equip an infinite-dimensional separable Hilbert space $\ell_{2}$ by such nonzero $\sigma$-finite Borel measures which are invariant with respect to everywhere dense vector subspaces and study duality between such measures and Baire category.

Section 1 contains constructions of nontrivial $\sigma$-finite Borel measures, which are defined in the infinite-dimensional separable Hilbert space $\ell_{2}$ and are invariant with respect to some everywhere dense vector subspaces. The duality between invariant Borel measures and Baire category in the classical Hilbert space $\ell_{2}$ is studied in Section 2. An idea applied in the process of proving of the main assertions allows us to obtain more general results for sufficiently large class of infinite-dimensional topological vector spaces.

1. Invariant Borel measures in classical Hilbert space $\ell_{2}$. Let $\mathbb{R}^{N}$ be the space of all sequences of real numbers equipped with the Tychonoff topology. Denote by $B\left(\mathbb{R}^{N}\right)$ the $\sigma$-algebra of all Borel subsets in $\mathbb{R}^{N}$.

Let $\left(a_{i}\right)_{i \in \mathbb{N}}$ and $\left(b_{i}\right)_{i \in \mathbb{N}}$ be sequences of real numbers such that

$$
(\forall i) \quad\left(i \in \mathbb{N} \rightarrow a_{i}<b_{i}\right)
$$

We put

$$
A_{n}=R_{0} \times \cdots \times R_{n} \times\left(\prod_{i>n} \Delta_{i}\right) \quad(n \in \mathbb{N}),
$$

where

$$
(\forall i) \quad\left(i \in \mathbb{N} \longrightarrow R_{i}=R, \Delta_{i}=\left[a_{i}, b_{i}[) .\right.\right.
$$

For an arbitrary natural number $i \in \mathbb{N}$, consider the Lebesgue measure $\mu_{i}$ defined on the space $R_{i}$ and satisfying the condition $\mu_{i}\left(\Delta_{i}\right)=1$. Denote by $\lambda_{i}$ the normed Lebesgue measure defined on the interval $\Delta_{i}$. 
For an arbitrary $n \in \mathbb{N}$, we denote by $v_{n}$ the measure defined by

$$
v_{n}=\prod_{1 \leq i \leq n} \mu_{i} \times \prod_{i>n} \lambda_{i}
$$

and by $\bar{v}_{n}$ the Borel measure in the space $\mathbb{R}^{N}$ defined by

$$
(\forall X) \quad\left(X \in B\left(\mathbb{R}^{N}\right) \longrightarrow \bar{v}_{n}(X)=v_{n}\left(X \cap A_{n}\right)\right) .
$$

The following assertion is valid.

LEMMA 1.1. For an arbitrary Borel set $X \subseteq \mathbb{R}^{N}$, there exists a limit

$$
v_{\Delta}(X)=\lim _{n \rightarrow \infty} \bar{v}_{n}(X)
$$

Moreover, the functional $\nu_{\Delta}$ is a nontrivial $\sigma$-finite measure defined on the Borel $\sigma$ algebra $B\left(\mathbb{R}^{N}\right)$.

Proof. First, observe that, for an arbitrary natural number $n$, the condition $A_{n} \subset$ $A_{n+1}$ is valid. By the property of $\sigma$-additivity of the measure $v_{n+1}$, we obtain

$$
\begin{aligned}
\bar{v}_{n+1}(X) & =v_{n+1}\left(X \cap A_{n+1}\right)=v_{n+1}\left(X \cap\left[A_{n+1} \backslash A_{n}\right] \cup A_{n}\right) \\
& =v_{n+1}\left[X \cap\left(A_{n+1} \backslash A_{n}\right)\right]+v_{n+1}\left(X \cap A_{n}\right) .
\end{aligned}
$$

Note that the restriction $\left.v_{n+1}\right|_{A_{n}}$ of the measure $v_{n+1}$ to the set $A_{n}$ coincides with the measure $v_{n}$.

Indeed, we have

$$
\begin{aligned}
v_{n+1}\left(A_{n} \cap X\right)= & \left(\prod_{1 \leq i \leq n+1} \mu_{i} \times \prod_{i>n+1} \lambda_{i}\right)\left(A_{n} \cap X\right) \\
= & \left\{\prod_{1 \leq i \leq n} \mu_{i} \times\left[\mu_{n+1}\left|\Delta_{n+1}+\mu_{n+1}\right|\left\{R \backslash \Delta_{n+1}\right\}\right] \times \prod_{i>n+1} \lambda_{i}\right\}\left(A_{n} \cap X\right) \\
= & \left(\prod_{1 \leq i \leq n} \mu_{i} \times \prod_{i>n} \lambda_{i}\right)\left(A_{n} \cap X\right) \\
& +\left(\prod_{1 \leq i \leq n} \mu_{i} \times\left(\mu_{n+1} \mid\left\{R \backslash \Delta_{n+1}\right\}\right) \times \prod_{i>n+1} \lambda_{i}\right)\left(A_{n} \cap X\right) \\
= & v_{n}\left(A_{n} \cap X\right) .
\end{aligned}
$$

Since for an arbitrary $n \in \mathbb{N}$, the inclusion $A_{n} \subset A_{n+1}$ holds, we have

$$
(\forall X) \quad\left(X \in B\left(\mathbb{R}^{N}\right) \longrightarrow v_{n}\left(A_{n} \cap X\right) \leq v_{n+1}\left(A_{n} \cap X\right)\right) .
$$

Hence there exists a limit $\lim _{n \rightarrow \infty} \bar{v}_{n}(X)$ which we denote by $v_{\Delta}(X)$.

The proof of the fact that the measure $v_{\Delta}$ is countably additive is trivial. 
Establish the following properties of $v_{\Delta}$.

(I) The measure $v_{\Delta}$ is nontrivial, since

$$
v_{\Delta}\left(\prod_{i \in \mathbb{N}} \Delta_{i}\right)=1 .
$$

(II) The measure $v_{\Delta}$ is $\sigma$-finite. Indeed, we have

$$
\mathbb{R}^{N}=\left(\mathbb{R}^{N} \backslash \bigcup_{n \in \mathbb{N}} A_{n}\right) \cup\left(\bigcup_{n \in \mathbb{N}} A_{n}\right) .
$$

Since $\mathbb{R}^{N} \backslash \bigcup_{n \in \mathbb{N}} A_{n} \in B\left(\mathbb{R}^{N}\right)$, by the definition of the measure $v_{\Delta}$ we have

$$
v_{n}\left(\mathbb{R}^{N} \backslash \bigcup_{k \in \mathbb{N}} A_{k}\right)=v_{n}\left(\left(\mathbb{R}^{N} \backslash \bigcup_{k \in \mathbb{N}} A_{k}\right) \cap A_{n}\right)=v_{n}(\varnothing)=0 .
$$

Since, for an arbitrary natural number $n \in \mathbb{N}$, the measure $\bar{v}_{n}$ is $\sigma$-finite, there exists a countable family $\left(B_{k}^{(n)}\right)_{k \in \mathbb{N}}$ of Borel measurable subsets of the space $\mathbb{R}^{N}$ such that

$$
\begin{array}{ll}
(\forall k) \quad & \left(k \in \mathbb{N} \longrightarrow \bar{v}_{n}\left(B_{k}^{(n)}\right)<+\infty\right) ; \\
(\forall n) \quad\left(n \in \mathbb{N} \longrightarrow A_{n}=\bigcup_{k \in \mathbb{N}} B_{k}^{(n)}\right) .
\end{array}
$$

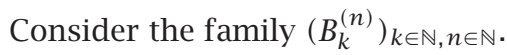

It is clear that

$$
(\forall k)(\forall n) \quad\left(k \in \mathbb{N}, n \in \mathbb{N} \longrightarrow v_{\Delta}\left(B_{k}^{(n)}\right)=\bar{v}_{n}\left(B_{k}^{(n)}\right)<+\infty\right) .
$$

On the other hand, we have

$$
\bigcup_{n \in \mathbb{N}} A_{n}=\bigcup_{n \in \mathbb{N}} \bigcup_{k \in \mathbb{N}} B_{k}^{(n)}
$$

that is,

$$
\mathbb{R}^{N}=\left(\mathbb{R}^{N} \backslash \bigcup_{n \in \mathbb{N}} A_{n}\right) \cup\left(\bigcup_{n \in \mathbb{N}, k \in \mathbb{N}} B_{k}^{(n)}\right) .
$$

The proof is completed.

REMARK 1.2. The measure $v_{\Delta}$ described in Lemma 1.1 can be regarded as an inductive limit of the family $(\bar{v})_{n \in \mathbb{N}}$ of invariant measures.

Recall that an element $h \in \mathbb{R}^{N}$ is called an admissible translation (in the sense of invariance) of the measure $v_{\Delta}$ if

$$
(\forall X) \quad\left(X \in B\left(\mathbb{R}^{N}\right) \longrightarrow v_{\Delta}(X+h)=v_{\Delta}(X)\right) .
$$

We define

$$
G_{\Delta}=\left\{h: h \in \mathbb{R}^{N}, h \text { is an admissible translation for } v_{\Delta}\right\} .
$$

It is easy to show that $G_{\Delta}$ is a vector subspace of the space $\mathbb{R}^{N}$. 
REMARK 1.3. The construction of the measure $v_{\Delta}$ belongs to Kharazishvili [1].

Our next theorem gives a representation of the algebraic structure of the vector subspace $G_{\Delta}$ of all admissible translations for $v_{\Delta}$.

THEOREM 1.4. The following conditions are equivalent:

$$
\begin{gathered}
g=\left(g_{1}, g_{2}, \ldots\right) \in G_{\Delta}, \\
\left(\exists n_{g}\right) \quad\left(n_{g} \in \mathbb{N} \longrightarrow \text { the series } \sum_{i=n_{g}}^{\infty} \ln \left(1-\frac{\left|g_{i}\right|}{b_{i}-a_{i}}\right) \text { is convergent }\right) .
\end{gathered}
$$

Proof. Assume that for an element $g=\left(g_{1}, g_{2}, \ldots\right) \in \mathbb{R}^{N}$, the condition (1.19) is satisfied. Then we have

$$
v_{\Delta}(\Delta+g)=v_{\Delta}(\Delta)=1
$$

On the other hand, we have

$$
\begin{aligned}
v_{\Delta}(\Delta+g) & =v_{\Delta}(\Delta+g) \\
& =v_{\Delta}\left(\prod _ { i \in \mathbb { N } } \left[a_{i}+g_{i}, b_{i}+g_{i}[)\right.\right. \\
& =\lim _{n \rightarrow \infty} \bar{v}_{n}\left(A_{n} \cap(\Delta+g)\right) \\
& =\lim _{n \rightarrow \infty}\left(\prod_{1 \leq i \leq n} \mu_{i} \times \prod_{i>n} \lambda_{i}\right)\left(\left(\prod_{1 \leq i \leq n} R_{i} \times \prod_{i>n}\left[a_{i}, b_{i}[) \cap \prod_{i \in \mathbb{N}}\left[a_{i}+g_{i}, b_{i}+g_{i}[)\right.\right.\right.\right. \\
& =\lim _{n \rightarrow \infty}\left(\prod _ { 1 \leq i \leq n } \mu _ { i } \left(\prod_{1 \leq i \leq n}\left[a_{i}+g_{i}, b_{i}+g_{i}[)\right) \times\left(\prod _ { i > n } \lambda _ { i } \left(\left[a_{i}+g_{i}, b_{i}+g_{i}[)\right)\right.\right.\right.\right. \\
& =\lim _{n \rightarrow \infty} \prod_{i>n} \lambda_{i}\left(\left[a_{i}, b_{i}\left[\cap \left[a_{i}+g_{i}, b_{i}+g_{i}[)=1 .\right.\right.\right.\right.
\end{aligned}
$$

We show that

$$
(\forall g) \quad\left(g=\left(g_{1}, g_{2}, \ldots\right) \in G_{\Delta} \longrightarrow \lim _{i \rightarrow \infty} \frac{\left|g_{i}\right|}{\left|b_{i}-a_{i}\right|}=0\right) .
$$

Indeed, if we assume the contrary, then there exist a countable subset $\left(n_{k}\right)_{k \in \mathbb{N}}$ of $\mathbb{N}$ and a positive real number $\epsilon>0$, such that

$$
(\forall k) \quad\left(k \in \mathbb{N} \rightarrow \frac{\left|g_{n_{k}}\right|}{b_{n_{k}}-a_{n_{k}}}>\epsilon\right) .
$$

Choose a number $m>0$ such that $\epsilon \cdot m>1$. Since $g \in G_{\Delta}$, we have

$$
m \cdot g=\left(m \cdot g_{1}, m \cdot g_{2}, \ldots\right) \in G_{\Delta}
$$


In view of the property of $\sigma$-additivity of the measure $\nu_{\Delta}$, we obtain

$$
v_{\Delta}(\Delta)=v_{\Delta}(\Delta+m \cdot g)=1
$$

But note that

$$
(\Delta+m \cdot g) \cap\left(\bigcup_{n \in \mathbb{N}} A_{n}\right)=\varnothing .
$$

Indeed, assume the contrary and take

$$
\left(x_{i}\right)_{i \in \mathbb{N}} \in(\Delta+m \cdot g) \cap\left(\bigcup_{n \in \mathbb{N}} A_{n}\right) .
$$

Then it is clear that, for the $n_{k}$ th coordinate, we have

$$
\begin{gathered}
\left(\exists k_{0}\right) \quad\left(k _ { 0 } \in \mathbb { N } \text { and } ( \forall k ) \quad \left(k \geq k_{0} \longrightarrow\left(a_{n_{k}}+m \cdot g_{n_{k}} \leq x_{n_{k}}<b_{n_{k}}+m \cdot g_{n_{k}}\right),\right.\right. \\
\left.\left.\left(a_{n_{k}} \leq x_{n_{k}}<b_{n_{k}}\right)\right)\right) .
\end{gathered}
$$

On the other hand, the validity of the condition

$$
(\forall k) \quad\left(k \in \mathbb{N} \rightarrow \frac{\left|g_{n_{k}}\right|}{b_{n_{k}}-a_{n_{k}}}>\epsilon\right)
$$

implies the validity of the relation

$$
(\forall k) \quad\left(k \in \mathbb{N} \longrightarrow m \cdot\left|g_{n_{k}}\right|>b_{n_{k}}-a_{n_{k}}\right),
$$

which shows that the intervals $\left[a_{n_{k}}, b_{n_{k}}\left[\right.\right.$ and $\left[a_{n_{k}}+m \cdot g_{n_{k}}, b_{n_{k}}+m \cdot g_{n_{k}}\right.$ [ have an empty intersection. Hence the condition $\lim _{i \rightarrow \infty}\left(\left|g_{i}\right| /\left(b_{i}-a_{i}\right)\right)=0$ holds.

From the validity of the condition $\lim _{i \rightarrow \infty}\left(\left|g_{i}\right| /\left(b_{i}-a_{i}\right)\right)=0$, we conclude that there exists a natural number $n_{\mathfrak{g}}$ such that

$$
(\forall i) \quad\left(i>n_{g} \rightarrow \frac{\left|g_{i}\right|}{b_{i}-a_{i}}<1\right)
$$

since

$$
(\forall i) \quad\left(i>n_{g} \longrightarrow \lambda_{i}\left(\left[a_{i}, b_{i}\left[\cap\left[a_{i}+g_{i}, b_{i}+g_{i}[)=\frac{b_{i}-a_{i}-\left|g_{i}\right|}{b_{i}-a_{i}}=1-\frac{\left|g_{i}\right|}{b_{i}-a_{i}}\right)\right. \text {. }\right.\right.\right.
$$

Keeping in mind that

$$
\lim _{p \rightarrow \infty} \prod_{i \geq n_{g}+p}\left(1-\frac{\left|g_{i}\right|}{b_{i}-a_{i}}\right)=1
$$

and considering the logarithms of both sides, we have

$$
\lim _{p \rightarrow \infty} \sum_{i \geq n_{g}+p} \ln \left(1-\frac{\left|g_{i}\right|}{b_{i}-a_{i}}\right)=0 .
$$

This means that the series $\sum_{i \geq n_{g}} \ln \left(1-\left|g_{i}\right| /\left(b_{i}-a_{i}\right)\right)$ is convergent. 
The validity of the implication $(1.19) \rightarrow(1.20)$ is proved.

Now we prove $(1.20) \rightarrow(1.19)$. Let $n_{g}$ be a natural number such that the series $\sum_{i \geq n_{g}} \ln \left(1-\left|g_{i}\right| /\left(b_{i}-a_{i}\right)\right)$ is convergent.

Consider an arbitrary element $X$ having the form

$$
X=B \times \prod_{i>n} \Delta_{i}
$$

where $B \in B\left(\mathbb{R}^{N}\right)(n \in \mathbb{N})$.

The sets of these forms generate the $\sigma$-algebra $B\left(A_{n}\right)$ of the space $A_{n}$, and the condition $B\left(A_{n}\right)=B\left(\mathbb{R}^{N}\right) \cap A_{n}$ holds. To prove the implication $(1.20) \rightarrow(1.19)$, it is sufficient to show the validity of the condition

$$
\begin{aligned}
v_{\Delta}(X+g)= & v_{\Delta}\left\{\left[\left(B \times \prod_{n+1 \leq i \leq n_{g}+n} \Delta_{i}\right)+\left(g_{1}, \ldots, g_{n_{g}}\right)\right] \times \prod_{i>n_{g}+n}\left[a_{i}+g_{i}, b_{i}+g_{i}[\}\right.\right. \\
= & \lim _{n \rightarrow \infty} \prod_{i=1}^{n_{g}+n} \mu_{i}\left(B \times \prod_{n+1 \leq i \leq n_{g}+n} \Delta_{i}\right) \\
& \times \prod_{i>n_{g}+n} \lambda_{i}\left(\left[a_{i}+g_{i}, b_{i}+g_{i}\left[\cap \left[a_{i}, b_{i}[)=v_{\Delta}\left(B \times \prod_{i>n} \Delta_{i}\right)\right.\right.\right.\right. \\
& \times \lim _{n \rightarrow \infty} \prod_{i>n_{g}+n}\left(1-\frac{\left|g_{i}\right|}{b_{i}-a_{i}}\right)=v_{\Delta}\left(B \times \prod_{i>n} \Delta_{i}\right)=v_{\Delta}(X) .
\end{aligned}
$$

We have used the well-known result from mathematical analysis

$$
\begin{gathered}
\text { (the series } \sum_{i \geq n_{g}} \ln \left(1-\frac{\left|g_{i}\right|}{b_{i}-a_{i}}\right) \text { is convergent) } \\
\Leftrightarrow \lim _{n \rightarrow \infty} \prod_{i \geq n_{g}+n}\left(1-\frac{\left|g_{i}\right|}{b_{i}-a_{i}}\right)=\ln 1 \\
\Leftrightarrow \lim _{n \rightarrow \infty} \prod_{i>n_{g}+n}\left(1-\frac{\left|g_{i}\right|}{b_{i}-a_{i}}\right)=1 .
\end{gathered}
$$

The proof is completed.

REMARK 1.5. Let $\mathbb{R}^{(N)}$ be the space of all finite sequences, that is,

$$
\mathbb{R}^{(N)}=\left\{\left(g_{i}\right)_{i \in \mathbb{N}} \mid\left(g_{i}\right)_{i \in \mathbb{N}} \in \mathbb{R}^{N}, \operatorname{card}\left\{i \mid g_{i} \neq 0\right\}<\mathrm{N}_{0}\right\}
$$

It is clear that, on the one hand, for an arbitrary compact infinite-dimensional parallelepiped $\Delta=\prod_{k \in \mathbb{N}}\left[a_{k}, b_{k}[\right.$, we have

$$
\mathbb{R}^{(N)} \subset G_{\Delta} .
$$


On the other hand, $G_{\Delta} \backslash \mathbb{R}^{(N)} \neq \varnothing$, since the element $\left(g_{i}\right)_{i \in \mathbb{N}}$ defined by

$$
(\forall i) \quad\left(i \in \mathbb{N} \rightarrow g_{i}=\left(1-\exp \left\{-\frac{b_{i}-a_{i}}{2^{i}}\right\} \times\left(b_{i}-a_{i}\right)\right)\right)
$$

belongs to the difference $G_{\Delta} \backslash \mathbb{R}^{(N)}$.

It is easy to show that the vector space $G_{\Delta}$ is everywhere dense in $\mathbb{R}^{N}$ with respect to the Tychonoff topology, since $\mathbb{R}^{(N)} \subset G_{\Delta}$.

In the sequel, we will need the following result.

THEOREM 1.6. In the separable Hilbert space $\ell_{2}$, there exists a $\sigma$-finite Borel measure $\lambda$ such that

(1) $\lambda\left(\Delta_{0}\right)=1$;

(2) a group $G_{\Delta_{0}}$ of all admissible translations of the measure $\lambda$ has the form

$$
\begin{aligned}
G_{\Delta_{0}}= & \left\{\left(c_{k}\right)_{k \in \mathbb{N}} \mid\left(c_{k}\right)_{k \in \mathbb{N}} \in \ell_{2},\right. \\
& \left.\left(\exists n_{p}\right)\left(n_{p} \in \mathbb{N} \longrightarrow \text { the series } \sum_{n=n_{p}}^{\infty} \ln \left(1-\left|c_{k}\right|(i+1)\right) \text { is convergent }\right)\right\},
\end{aligned}
$$

where $\Delta_{0}=\prod_{i \in \mathbb{N}}[0 ; 1 /(i+1)[$.

Proof. According to Suslin's theorem we have $B\left(\ell_{2}\right) \subseteq B\left(\mathbb{R}^{N}\right)$. Now the proof of Theorem 1.6 can be obtained easily if we put

$$
(\forall X) \quad\left(X \in B\left(\ell_{2}\right) \Longrightarrow \lambda(X)=v_{\Delta_{0}}\left(\ell_{2} \cap X\right)\right) .
$$

2. Duality of measure and category in the infinite-dimensional separable Hilbert space $\ell_{2}$. In this section, we continue our discussion of some properties of invariant measures in the infinite-dimensional separable Hilbert space $\ell_{2}$ and study the question of the duality between the Baire category and the above-constructed measure $\lambda$.

The following definitions are important for our investigation.

Let $(E, T)$ be a nonempty topological vector space. Denote by $B(E)$ the Borel $\sigma$ algebra of subsets of the space $E$, generated by the topology $T$. Consider a nontrivial Borel measure $\mu$ defined on the $\sigma$-algebra $B(E)$. A subset $X \subseteq E$ is called small in the sense of measure if $\mu^{*}(X)=0$. Analogously, a subset $Y \subseteq E$ is called small in the sense of category if it is the first category set in the topological space $(E, T)$. Further, let $P$ be a such sentence in formulation of which the notions of measure zero and of the first category are used. We say that the duality between the measure $\mu$ and the Baire category is valid with respect to the sentence $P$ if the sentence $P$ is equivalent to the sentence $P^{*}$ obtained from the sentence $P$ by interchanging the notions of the above small sets. We also say that strict duality between the measure $\mu$ and Baire category is valid if the duality between the measure $\mu$ and the Baire category is valid for all the above $P$ sentences formulated only by using the notions of measure zero, of first category and of purely set-theoretical notions. 
The following result is known as the Erdős-Sierpiński duality principle.

THEOREM 2.1 (duality principle). If the continuum hypothesis is true, then the strict duality between a linear Lebesgue measure and the Baire category of the real axis $\mathbb{R}$ is valid.

The proof of Theorem 2.1 can be found, for example, in [4].

Using the same argument applied in the process of the proving of Theorem 2.1 (see [4, pages 129-131]), it is easy to conclude that if the continuum hypothesis is true, then the strict duality between the measure $\lambda$ and Baire category of $\ell_{2}$ is valid also.

Here we apply the well-known method to establish one important property of Baire second category subsets in the infinite-dimensional separable Hilbert space $\ell_{2}$.

THEOREM 2.2. For an arbitrary second category Baire subset $X \subseteq \ell_{2}$, there exists a positive number $\delta>0$ such that

$$
(\forall x) \quad\left(x \in \ell_{2},\|x\|<\delta \longrightarrow(X+x) \cap X \neq \varnothing\right) .
$$

Proof. Since the set $X$ has the Baire property, there exist an open subset $G \subseteq \ell_{2}$ and a first category subset $P \subseteq \ell_{2}$ such that the equality

$$
X=G \Delta P
$$

is fulfilled.

Evidently, there exists an open nonempty ball $B \subseteq G$.

Note that the inclusion

$$
[(x+B) \cap B] \backslash[P \cup(x+P)] \subseteq(x+X) \cap X
$$

holds for arbitrary $x \in \ell_{2}$. If $\|x\|<\operatorname{diam}(B)$, then the set, the left-hand side of (2.3), is a nonempty open set minus a first category set.

Using the well-known Baire theorem, we complete the proof of Theorem 2.2.

REMARK 2.3. The method considered in the proof of Theorem 2.2 was worked out and applied by many authors, for example, Oxtoby who establishes an analogous result for linear Baire second category subsets in $\mathbb{R}$ (cf. [4]).

The following simple result (which is however important from the viewpoint of applications) is also essentially due to Steinhaus.

THEOREM 2.4. Let $X$ be an arbitrary linear Borel subset in $\mathbb{R}$ with a positive Lebesgue measure. Then there exists a positive number $\delta$ such that the condition

$$
(\forall x) \quad(x \in \mathbb{R},|x|<\delta \rightarrow(x+X) \cap X \neq \varnothing)
$$

holds.

The proof of Theorem 2.4 can be found in [4].

The next theorem plays the main role in our further consideration.

THEOREM 2.5. In the infinite-dimensional separable Hilbert space $\ell_{2}$, there exists a Borel subset $Y \subset \ell_{2}$ with $\lambda(Y)>0$ such that

$$
(\forall \delta) \quad(\delta>0 \longrightarrow(\exists y)(\|y\|<\delta \longrightarrow Y \cap(Y+y)=\varnothing)) .
$$


Proof. Let

$$
Y \equiv \Delta_{0}=\prod_{i \in \mathbb{N}}\left[0, \frac{1}{i+1}[.\right.
$$

For an arbitrary positive real number $\delta>0$, denote by $n_{\delta}$ a natural number such that

$$
\sum_{i=n_{\delta}}^{\infty} \frac{1}{(i+1)^{2}}<\delta^{2} .
$$

Assume that

$$
\begin{aligned}
& (\forall k) \quad\left(1 \leq k<n_{\delta} \longrightarrow h_{k}=0\right), \\
& (\forall k) \quad\left(k \geq n_{\delta} \longrightarrow h_{k}=\frac{1}{k+1}\right)
\end{aligned}
$$

It is clear that $h=\left(h_{k}\right)_{k \in \mathbb{N}} \notin G_{\Delta_{0}},\|h\|<\delta$, and $\Delta_{0} \cap\left(\Delta_{0}+h\right)=\varnothing$. Theorem 2.5 is proved.

Summarizing all the above results, we obtain the following statement.

THEOREM 2.6. The duality between the measure $\lambda$ and the Baire category with respect to the sentence $P_{0}$, where

$$
\begin{aligned}
& P_{0}=(\forall X) \quad\left(X \subseteq \ell_{2}, X\right. \text { is a Baire subset of second category } \\
&\longrightarrow(\exists \delta)(\delta>0 \longrightarrow(\forall x)(\|x\|<\delta \longrightarrow X \cap(X+x) \neq \varnothing))),
\end{aligned}
$$

is not valid.

REMARK 2.7. By Remark 2.3 and Theorem 2.4, it is easy to obtain the validity of the duality between the linear Lebesgue measure and the Baire category with respect to the sentence $P_{0}$ in $\mathbb{R}$. This result is essentially due to Oxtoby and may be called Oxtoby duality principle in $\mathbb{R}$ (cf. [4]).

REMARK 2.8. Theorem 2.6 states that an analogy of the Oxtoby duality principle is not valid for the measure $\lambda$ and the Baire category in the infinite-dimensional separable Hilbert space $\ell_{2}$.

There are also several important works devoted to the solution of analogous problems in various topological vector spaces (cf. [2, 3] and others).

The following notion is frequently useful in studying various questions of measure theory.

We say that the measure $\mu$ defined in a topological vector space $(E, T)$ satisfies the axiom of Steinhaus if the following condition:

$(\forall X) \quad(X \in \operatorname{dom}(\mu), \mu(X)<\infty$

$\longrightarrow(\forall \epsilon)\left(\epsilon>0 \longrightarrow\left(\left(\right.\right.\right.$ there exists a neighborhood $V_{\epsilon}$ of the zero vector 0$)$,

$$
\left.\left.\left.\left((\forall h)\left(h \in V_{\epsilon} \longrightarrow \mu((X+h) \triangle X)<\epsilon\right)\right)\right)\right)\right)
$$

holds. 
THEOREM 2.9. The measure $\lambda$ does not satisfy the axiom of Steinhaus.

Proof. Assume the contrary. Then for the set $\Delta_{0}$ and for the number $\epsilon=1 / 2$, there exists a number $\delta>0$ such that

$$
(\forall x)\left(\|x\|<\delta \rightarrow \lambda\left(\left(\Delta_{0}+x\right) \triangle \Delta_{0}\right)<\frac{1}{2}\right) .
$$

Consider the element $h=\left(h_{k}\right)_{k \in \mathbb{N}}$ constructed in Theorem 2.5. Since $\|h\|<\delta$, $\left(\Delta_{0}+h\right) \cap\left(\bigcup_{n \in \mathbb{N}} A_{n}\right)=\varnothing$, and the measure $\lambda$ is concentrated on the set $\bigcup_{n \in \mathbb{N}} A_{n}$, where $A_{n}$ is defined in Section 1 for $\Delta=\Delta_{0}$, we have $\lambda\left(\left(\Delta_{0}+h\right) \Delta \Delta_{0}\right)=\lambda\left(\Delta_{0}\right)=1$. This contradicts the condition

$$
\lambda\left(\left(\Delta_{0}+h\right) \triangle \Delta_{0}\right)<\frac{1}{2}
$$

Thus, Theorem 2.9 is proved.

REMARK 2.10. We must say that the analogies of Theorems 2.6 and 2.9 are valid for an arbitrary nontrivial $\sigma$-finite Borel measure and Baire category defined in infinitedimensional Polish topological vector space, but this question will not concern us here.

EXAMPLE 2.11. Define the measure $\mu_{0}$ by

$$
(\forall B) \quad\left(B \in B\left(\ell_{2}\right) \longrightarrow \mu_{0}(B)= \begin{cases}\infty, & \text { if } \mathbf{B} \text { is of second category, } \\ 0, & \text { if } \mathbf{B} \text { is of first category) } .\end{cases}\right.
$$

It is proved that, on the one hand, the measure $\mu_{0}$ satisfies Suslin's property and is invariant with respect to the vector space $\ell_{2}$ (see [3]). On the other hand, using Theorem 2.2, we conclude that the measure $\mu_{0}$ (unlike the measure $\lambda$ ) satisfies

$$
\begin{aligned}
(\forall X) \quad\left(X \in B\left(\ell_{2}\right), \mu(X)>0\right. \\
\quad \longrightarrow(\exists \delta)(\delta>0 \longrightarrow(\forall h)(\|h\|<\delta \longrightarrow(X+h) \cap X \neq \varnothing))) .
\end{aligned}
$$

This means that the duality between the measure $\mu_{0}$ (which is not $\sigma$-finite) and the Baire category, with respect to the property $P_{0}$, is valid in the separable Hilbert space $\ell_{2}$. Also note that the measure $\mu_{0}$ satisfies the axiom of Steinhaus.

REMARK 2.12. Clearly, it is not possible to define, in the space $\ell_{2}$, a translationinvariant nontrivial $\sigma$-finite Borel measure. But if we ignore the condition of $\sigma$ finiteness, then in some consistent system of axioms, the construction of such Borel measures is possible (cf. [5]). In connection with the above results, one can pose the problem of the validity of the duality between the translate-invariant Borel measure and the Baire category with respect to the property $P_{0}$ in the infinite-dimensional separable Hilbert space $\ell_{2}$.

\section{REFERENCES}

[1] A. B. Kharazishvili, Invariant measures in Hilbert space, Soobshch. Akad. Nauk Gruzin. SSR 114 (1984), no. 1, 45-48. 
[2] _ Topologicheskie aspekty teorii mery, Naukova Dumka, Kiev, 1984 (Russian).

[3] _ On Borel measures in space $R^{\alpha}$, Ukrainian Math. J. 40 (1988), no. 5, 665-668.

[4] J. C. Oxtoby, Measure and Category, 2nd ed., Graduate Texts in Mathematics, vol. 2, Springer-Verlag, New York, 1980.

[5] G. R. Pantsulaia, The construction of invariant measures in the non-separable Banach space $l^{\infty}$, Georgian Technical University Press 430 (2000), no. 2, 18-20.

Gogi Pantsulaia: Department of Higher Mathematics, Georgian Technical UniverSITY, 77 KostaVA STREET, TBILISI 380043, GeORGIA

E-mail address: gogi@gtu.edu.ge 


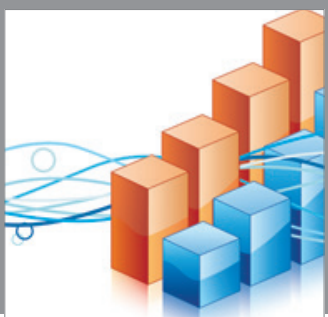

Advances in

Operations Research

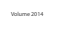

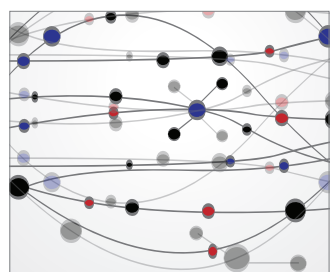

\section{The Scientific} World Journal
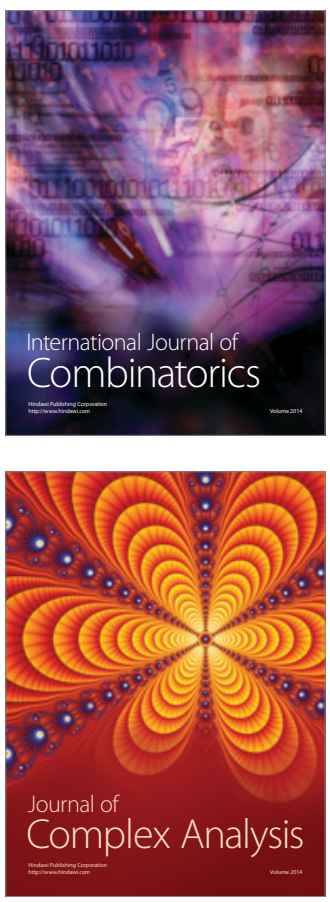

International Journal of

Mathematics and

Mathematical

Sciences
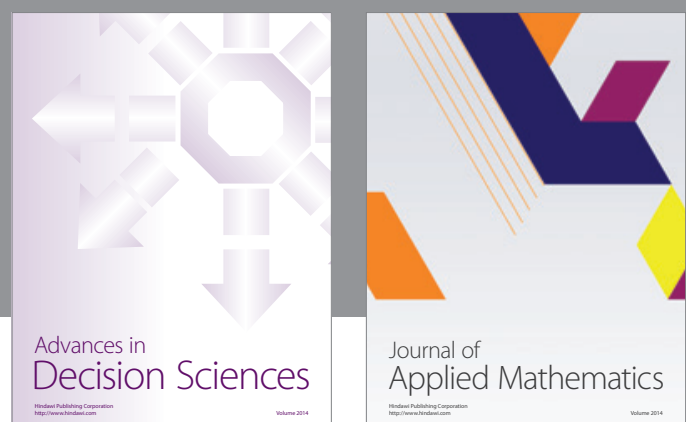

Journal of

Applied Mathematics
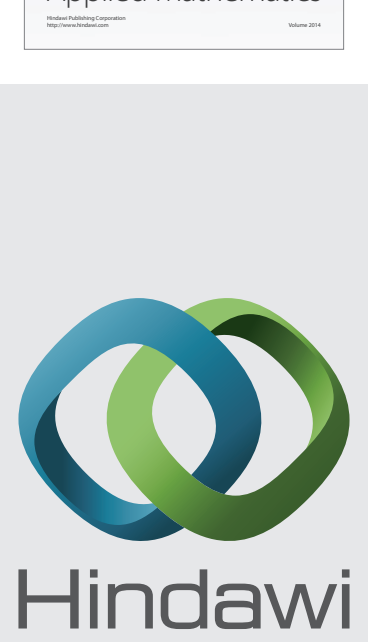

Submit your manuscripts at http://www.hindawi.com
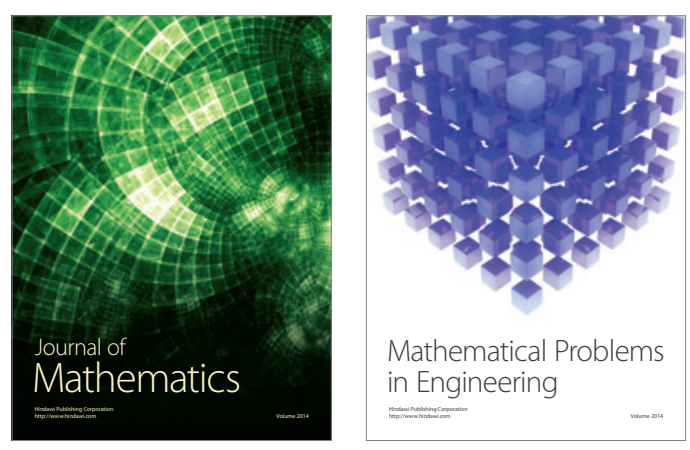

Mathematical Problems in Engineering
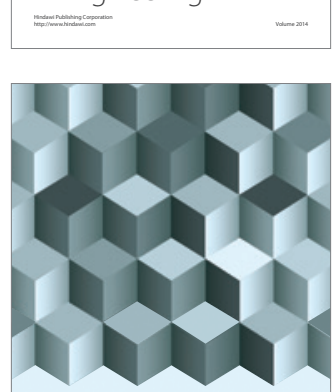

Journal of

Function Spaces
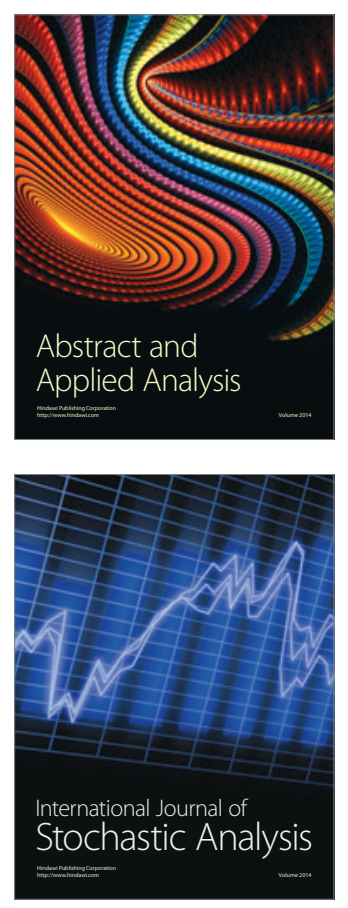

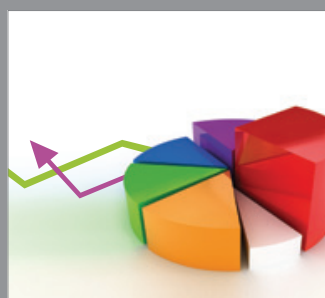

ournal of

Probability and Statistics

Promensencen
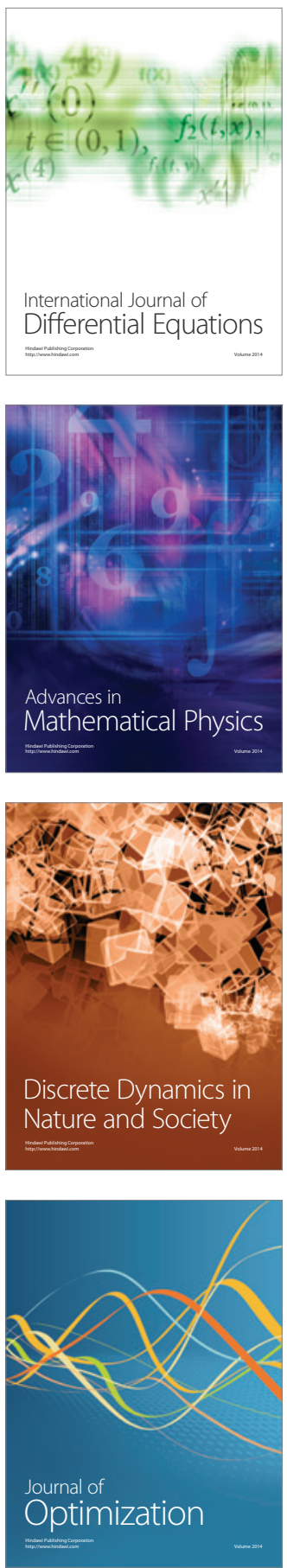